\title{
The Current State of Recurrent Laryngeal Nerve Monitoring for Thyroid Surgery
}

\author{
Gianlorenzo Dionigi - Che-Wei Wu • Davide Lombardi - Remo Accorona • \\ Anna Bozzola $\cdot$ Hoon Yub Kim $\cdot$ Feng-Yu Chiang $\cdot$ Maurizio Bignami • \\ Paolo Castelnuovo $\cdot$ Piero Nicolai
}

Published online: 17 December 2013

(c) Springer Science+Business Media New York 2013

\begin{abstract}
There is an increasing interest in intraoperative nerve monitoring (IONM), and numerous institutions have begun to perform monitored thyroidectomies. Attitudes have changed with the introduction of non-invasive devices, the publication of trials and guidelines defining standards, structured courses and descriptions of legal implications. The use of IONM helps to identify the nerve and give an objective evaluation of its function during the dissection. Recently, continuous IONM was introduced; it is a promising tool for early recognition of recurrent laryngeal nerve stress. This paper describes current issues in IONM.
\end{abstract}

Keywords Recurrent laryngeal nerve $\cdot$ Nerve monitoring · Guidelines · Research $\cdot$ Stage thyroidectomy $\cdot$ Informed consent

G. Dionigi $(\square)$

First Division of General Surgery, Department of Surgical

Sciences and Morphology, University of Insubria, Varese, Italy

e-mail: gianlorenzo.dionigi@uninsubria.it

C.-W. Wu $\cdot$ F.-Y. Chiang

Department of Otolaryngology, Head and Neck Surgery

Kaohsiung Medical University Hospital, Kaohsiung Medical

University, Kaohsiung, Taiwan

D. Lombardi · R. Accorona · A. Bozzola $\cdot$ P. Nicolai

Department of Otorhinolaryngology, Head and Neck Surgery,

University of Brescia, Spedali Civili of Brescia, Brescia, Italy

H. Y. Kim

Department of Surgery, Korea University College of Medicine,

Seoul, Korea

M. Bignami · P. Castelnuovo

Department of Otorhinolaryngology, University of Insubria,

Varese, Italy

\section{Introduction: State of the Art}

Intraoperative neuromonitoring (IONM) has been applied as an adjunct to standard visual identification of the recurrent laryngeal nerve (RLN) to help prevent nerve paralysis $[1 \bullet, 2 \bullet \bullet, 3]$. In the last decade, IONM has gained widespread acceptance among surgeons (Fig. 1), and much research has been conducted to solve common pitfalls and investigate implications and new applications of IONM [2••, 3-15] (Fig. 2).

De facto, as correctly noted in recent publications $[2 \bullet \bullet$, 4-15], the main aims of IONM are as follows:

(1) Early definitive localization of the nerve, thus avoiding excessive manipulation and damage to RLN and possible extralaryngeal branch(es) or anatomic variants (i.e. the nonrecurrent laryngeal nerve);

(2) Confirmation of RLN visual identification (preventing visual RLN misidentification);

(3) Evaluation of the laryngeal nerve function before, during and after dissection, with objective confirmation of the neurophysiological integrity on the first side before approaching the contralateral side.

Most of the existing studies have shown that although there is a reduction in the rate of RLN paralysis with the use of standardized IONM compared with the gold standard of nerve visualization alone, the difference between the two methods is not statistically significant $\left[3,16^{\bullet \bullet}\right]$. Only one prospective randomized study has shown significant benefit for the reduction of the temporary palsy rate with IONM $[17 \bullet \bullet]$.

The uncertainty in the literature concerning the merit of IONM to reduce overall RLN paralysis led to the analysis of other possible benefits; for example, in the case of an inexperienced surgeon, typelrisk of thyroid disease treated, 


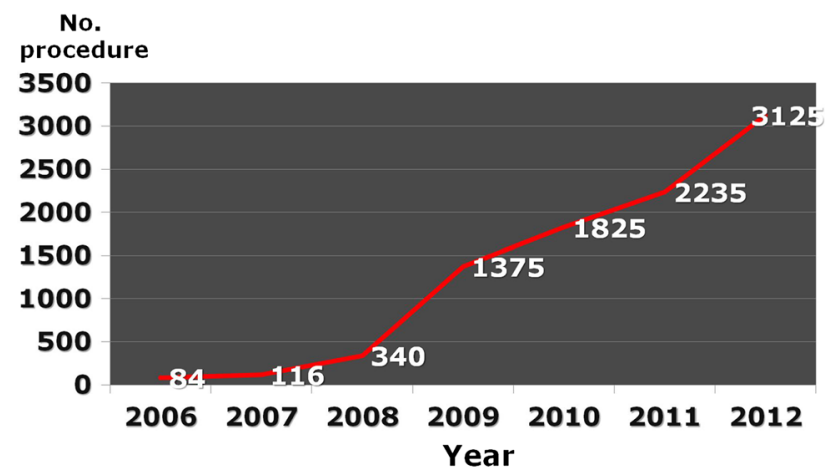

Fig. 1 Increasing prevalence of use of intraoperative neuromonitoring (IONM) in Italy. Source data: Inomed, Langer, Medtronic, Italy manufacturers' sales data

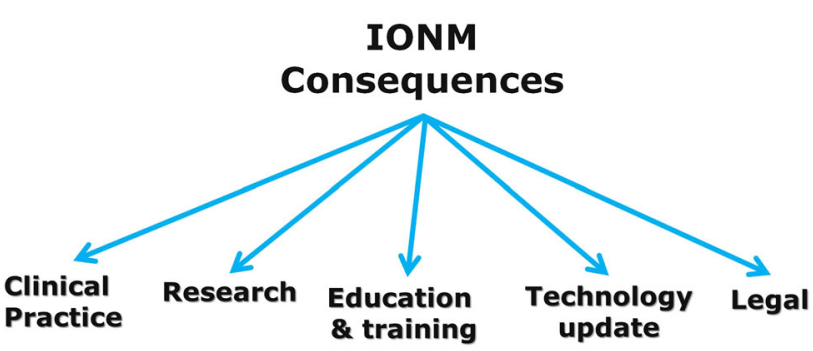

Fig. 2 Implications and consequences of IONM

surgical procedure and strategy (i.e. central compartment neck dissection (CCND) and to prevent bilateral RLN injury).

Dralle et al. $[3,18]$ demonstrated that the rates of nerve paralysis correlate with the surgical experience, and that a minor surgical experience is associated with a lower rate of paralysis when the nerve is identified both visually and with the aid of IONM.

Because both reoperation and first thyroid operations with an atypical course and anatomy of the recurrent nerve are associated with an increased risk of paralysis [3, 19••], it must be assumed that even in situations of this type, neuromonitoring presents an advantage because it allows a better representation of the course of nerve. The experienced surgeon must remember that some surgeries are difficult not only because of the thyroid disease being treated (e.g. high-risk procedures such as Graves' disease, cancer and re-do surgery), but also because of atypical anatomy of the neck and the RLN (non-RLN, RLN ramification, relationship with the inferior thyroid artery, Berry's ligament and Zuckerkandl tubercle and nerve dislocation) $[3,19 \bullet \cdot$. These situations cannot be identified beforehand, preoperatively.

In a recent study by Barczynski, IONM decreased the incidence of transient RLN paresis in repeat thyroid operations compared with nerve visualization alone [20••]. In detail, transient and permanent RLN injuries were found respectively in 2.6 and $1.4 \%$ nerves with IONM, versus 6.3 and $2.4 \%$ nerves without IONM $(p=0.003$ and $p=0.202$, respectively) $[20 \bullet \cdot$.

A more recent retrospective study of a large cohort of patients demonstrated that adding CCND to total thyroidectomy does not increase the risk of transient or permanent RLN palsy [21]. Moreover, Chiang et al. [22] reported that in 101 patients, extensive RLN dissection did not result in a decrement of neurotransmission. Therefore, the role of IONM during primary CCND, either elective or therapeutic, remains controversial; nevertheless, the possibility of better identification of the nerve, especially in the presence of the aforementioned anatomic variants, and objective evaluation of the nerve's status before approaching the contralateral site that minimizes the risk of bilateral nerve palsy, remain two important factors that cannot be neglected. In contrast, when performing a revisional CCND, IONM can be extremely useful, because it may ease the identification of the RLN in a surgical field that lacks anatomic landmarks and may have abundant scar tissue. In this scenario, the mapping of the area where the nerve is supposed to be may avoid a dangerous "blind" dissection [23]. As for CCND, once thyroidectomy has been completed, all the important anatomic structures, including the RLN, should have been clearly identified and preserved; thus, CCND should not entail an increased risk of misidentification of the nerve per se. In contrast, the increased manipulation of the RLN associated with CCND could be expected to compromise its function (Fig. 3).

\section{Technology Perspectives}

\section{Continuous IONM ( $C$-IONM)}

Currently, the intermittent IONM (I-IONM) mode of application presents relevant limits (Table 1) [24]. With I-IONM, the functional integrity of the RLN is limited to the short interval of direct nerve stimulation [24]. Via $I$-IONM, the wholeness of the laryngeal nerve is limited merely to the site of direct nerve stimulation: in proximal neurogenic lesions of the RLN, distal stimulation near the larynx may produce a false negative "normal" IONM signal [24]. Thus, the RLN is still at risk for damage during thyroidectomy (a) proximal to the site of the surgeons' stimulation and/or $(b)$ during the time gap between two nerve stimulations. De facto, $I$-IONM tests the integrity of the RLN just following a thyroid dissection, allows the evaluation of the RLN solely at the moment of stimulation, and detects RLN injury merely after insult occurs [24].

To overcome these $I$-IONM limits, a $C$-IONM technology has been proposed. [25-28] $C$-IONM represents an 


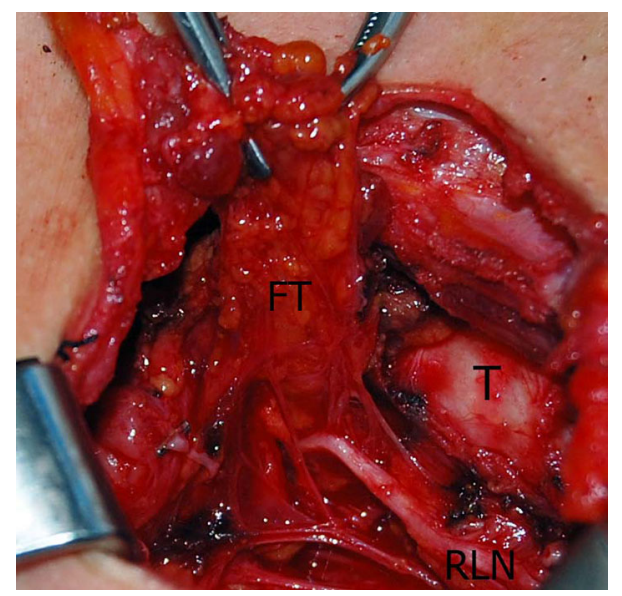

Fig. 3 Central compartment neck dissection (CCND) on the left side. The dissection of the recurrent laryngeal nerve (RLN) should be performed with blunt instruments, trying to exert a minimal traction on the fibro-adipose tissue (FT). (T: trachea). IONM is useful to test the function of RLN during and after CCND

Table 1 Limits of intermittent IONM

1. RLN palsy still occurs

2. Need for standardized and well-trained use to avoid pitfalls

3. Knowledge of most-common pitfalls

4. Ability to use troubleshooting algorithms

5. IONM does not replace clinical judgment

6. Relatively low positive predictive value

7. Cost-effectiveness is still not evaluated

8. Need for further research focused on neurophysiology of the RLNs

9. IONM of the external branch of the superior laryngeal nerve 10. Assessment of posterior branch of RLN

11. Thyroidectomy in local anesthesia

automatic stimulation of the vagus nerve (VN) using a software-based reassessment of the change in electromyographic endotracheal (EMG) amplitude and latency during surgery. According to preliminary observations, this mode of IONM application provides the surgeon with real-time visual and acoustic feedback about the current RLN conductivity, allows testing for the integrity of the RLN throughout the dissection, and allows steady evaluation of the RLN [25-28].

The recent introduction of $C$-IONM includes several considerations.

$C$-IONM might perceive imminent and/or increasing RLN risk intraoperatively. In principle, in cases of weak and decreased EMG signal, the surgeon may react early intraoperatively to RLN stress, and RLN injury may be reversible. Theoretically, the surgeon may avoid eventual further injury to the RLN, and the nerve will be restored by the early relief of trauma. This may occur in neuropraxia, but not in the case of a more significant disruption, acute, or complete division of a nerve, as in cases of neurotmesis or axonotmesis (i.e. RLN section or in case of thermal injury). According to recent observation [25-28], RLN traction injury is still the most common cause of RLN injury. $C$-IONM is useful for preventing imminent traction injury by detecting progressive decreases in EMG amplitude combined with progressive latency increases. As recently described by Schneider et al. [27], to facilitate the interpretation of clinically relevant quantitative EMG signals, adverse EMG events should be categorized based both (combined and multiple) on changes of evocated signal amplitude and latency, consisting of a $>50 \%$ decrease in amplitude paralleled by a $>10 \%$ increase in latency.

Further clinical research, especially in neurophysiology and neuropathology, is necessary for qualitative and quantitative analyses and interpretations of the change in signal obtained with $C$-IONM before the procedure can be widely used on patients [24]. These data are essential to the surgeon's intraoperative decision-making process.

The quality of the EMG signal is fundamental. A critical evaluation of the currently available neuromonitoring devices is essential for the development and introduction of this new IONM application method. Moreover, under EMG endotracheal surface electrodes, it is difficult to tell whether the change in EMG amplitude is caused by the change in contact between the electrodes and vocal cords or by true nerve injury. In addition, an EMG signal from $C$-IONM application might be limited by the type of anesthesia, the manipulation of the trachea and the vagal nerve electrode dislocation (acute signal loss $=$ electrode dislocation) [24]. The stimulating electrodes must be configured in a manner that protects against dislocation and changes in their distance from the nerve during surgical manipulation within the surgical site.

The VN electrode for $C$-IONM should be versatile $[4,6]$. The location of the $\mathrm{VN}$ in relation to the common carotid artery (CCA) and internal jugular vein (IJV) is classified as $A$ (anterior), $P$ (posterior), $P J$ (posterior to IJV), or $P C$ (posterior to the CCA) [6]. Most vagal nerves lay in the posterior region of the carotid sheath, in the groove between these two vessels (95\%) [6]. The $P$ location of the $\mathrm{VN}$ is the most common configuration observed on either side, followed by the $P C(15 \%)$ and $P J(8 \%)$ locations. Overall, less than $5 \%$ of $A$ location cases are observed $[4,6]$. Moreover, the medial location of the CCA and the anterolateral or lateral location of the IJV are the most common configurations in the carotid sheath. Few cases of medial IJV position are observed. Tortuosity, kinking, or coiling of the extracranial carotid arteries may be observed with advancing age $[4,6]$.

It is equally essential that the safety of a new procedure is established before it is widely used on patients. With 
I-IONM, a lesser and partial dissection of the carotid sheath and VN is used: the VN is exposed by dissecting the carotid sheath from just a $1-\mathrm{cm}$ pouch; thus, not all of the carotid sheath is completely or routinely dissected. Moreover, when $I$-IONM is used to expedite VN identification or when the surgeon is not confident about carotid sheath dissection (in case of fatty areolar tissue, reoperative surgery, large goiter, hostile neck, endoscopic thyroidectomy or gross lateral metastatic lymph nodes that may displace the VN), VN identification and stimulation may be facilitated simply by increasing the amplitude to $2-3 \mathrm{~mA}$ with the probe on the carotid sheath without dissecting the neck vessel sheath [4]. Nevertheless, most $C$-IONM methods require dissecting the $\mathrm{VN}$ circumferentially $\left(360^{\circ}\right)$ to place the VN electrode on it. This procedure can be difficult, time-consuming, or even harmful to the VN and carotid sheath while the electrode is positioned during surgery and when it is removed. The electrode placement should be tension-free. Experimental histologic evaluation on the VN after probe positioning and repeated stimulation is necessary. The systemic safety of $C$-IONM has been already evaluated [4].

Finally, we would like to again emphasize the importance of $I$-IONM: traditional manual stimulation should still be used in combination with $C$-IONM. $C$-IONM seems to be a technological improvement. Likely, $C$-IONM via vagal nerve stimulation should enhance the standardization process, intraoperative RLN information, documentation, protection, training, and research in modern thyroid surgery.

\section{IONM in Endoscopic Thyroidectomy}

There have been four reports describing the efficacy of IONM in endoscopic thyroidectomy [29, 30••, 31, 32]. Kandil et al. [29] first reported the retrospective data for IONM use in minimally invasive video-assisted thyroidectomy, and concluded that IONM could be a very useful adjunct for RLN identification during endoscopic thyroid surgery. However, their method of IONM is not limited to the standardized IONM technique, in which the essential IONM procedure is vagal stimulation prior to the direct identification of RLN [1••, 9, 29]. The standardization of the IONM technique covers a fundamental technical aspect of thyroid surgery and endoscopic thyroidectomy. In 2012, Dionigi et al. [30••] reported a study of RLN injury during video-assisted thyroidectomy (VAT). They described the standardized IONM technique used in VAT surgery. The technique is composed of six steps [30•*]: (1) preoperative laryngoscopy; (2) VN stimulation before thyroidectomy; (3) RLN stimulation upon initial identification; (4) RLN stimulation at the end of thyroid dissection and complete hemostasis; (5) VN stimulation after complete thyroidectomy and hemostasis; and (6) Postoperative laryngoscopy $[1 \bullet \bullet, 9,30 \bullet \cdot$. The fundamental steps of IONM during endoscopic thyroidectomy are the same as those used during conventional open surgery, and this technique should not be different in other approaches to endoscopic thyroidectomy.

Lang et al. [31] reported a retrospective study of IONM during gasless transaxillary endoscopic thyroidectomy and robotic-assisted thyroidectomy. This study demonstrated the technical feasibility of using a conventional open-nerve stimulator probe in both gasless transaxillary endoscopic thyroidectomy and robotic-assisted thyroidectomy [31]. They also compared two nerve stimulation techniques: direct RLN stimulation and indirect stimulation via the VN [31]. The result of this study showed that indirect stimulation via the $\mathrm{VN}$ produced more reliable and accurate IONM results than direct RLN stimulation [31]. This result supports the assertion that the standardized IONM technique is essential.

In endoscopic or robotic surgery, the electromyographic endotracheal (EMG) tube is identical to that used in conventional open thyroidectomy. However, a conventional probe cannot be used. Instead, there are two alternative methods for stimulating nerves. First, some hospitals use a flex wire stim probe (Fig. 4). This wire goes between the cannula and skin to access the vagus or laryngeal nerves. The wire probe stimulates the nerve via an endoscopic or robotic grasper. Generally, the NIM stimulation unit (NIM Response 3.0 System $^{\circledR}$, Medtronic Xomed, Jacksonville, FL, USA) is linked to a conventional probe or wire probe. In our hospital, we connect the NIM stimulation unit to the endoscopic monopolar electrocauterization hook. This second method is convenient because it allows the operator to stimulate the nerve without grasping the wire probe, and this procedure makes it easy to switch between cauterization and stimulation.

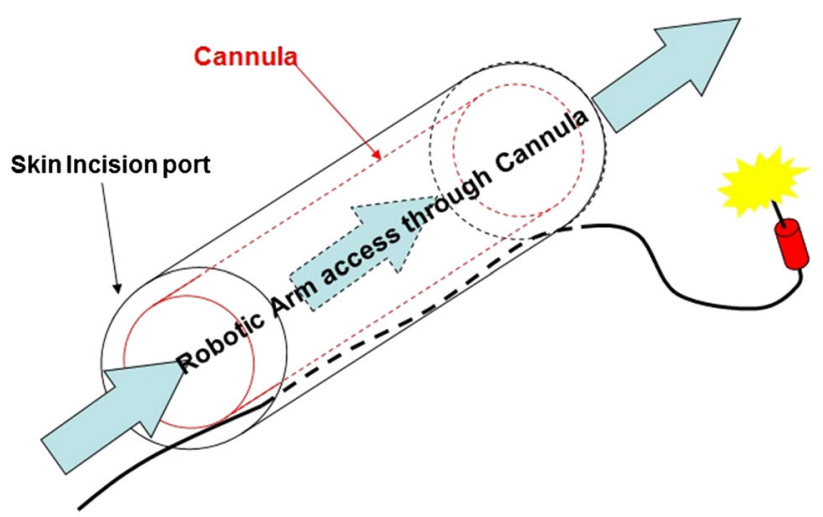

Fig. 4 Diagram of flex wire stim probe. The probe is connected with electronic stimulator through flexible wire and goes between the cannula and skin 
In 2009, Dionigi et al. [32] reported a prospective, randomized case-control study of neuromonitoring in video-assisted thyroidectomy using a standardized IONM technique. To our knowledge, this is the only randomized controlled study of IONM in endoscopic thyroidectomy. The authors successfully showed the technical feasibility and safety of IONM in VAT [32]. The incidences of temporary RLN injury were 2.7 and $8.3 \%$ in the IONM and control group, respectively [32]. The authors concluded that IONM enables surgeons to feel more comfortable with their approach to VAT [29, 30••, 31, 32]; however, they did not show a significant reduction in postoperative complications among the IONM group [32]. Thus, larger series with prospective, randomized, controlled studies will be needed to prove the benefit of IONM for RLN injury prevention.

In endoscopic thyroidectomy, standardized IONM is as feasible and safe as conventional open thyroidectomy.

\section{Issues Surrounding IONM}

The introduction of IONM has implications for preoperative and postoperative patient management.

\section{Preoperative Management}

Preoperative management may include adequate patient information and a specific informed consent. Thus, a proposed informed consent may describe the possible consequences of thyroidectomy, the risk of the operation and the consequences of IONM. Therefore, the patient must be informed preoperatively of the possibility of undergoing thyroidectomy in two phases if loss of signal (LOS) to the first side occurs. If a total thyroidectomy is planned, the possibility of learning intraoperatively that one nerve is injured aids in the decision about whether to go to the other side. IONM allows the surgeon to stage contralateral surgery if RLN damage is diagnosed, thereby avoiding the potential for bilateral vocal cord paralysis [33].

De facto, possible consequences of a LOS of the RLN on the first side is helpful in considering optimal contralateral surgery timing. With LOS, the surgeon must consider that the ipsilateral nerve is injured at least temporarily, and can determine whether it is important enough and in the patient's best interest to perform surgery on the contralateral side that day, given the new intraoperative information about ipsilateral paralysis [33]. According to the Goretzki group [34॰•], a failed IONM stimulation of RLN after resection of the first thyroid lobe is specific enough to reconsider the surgical strategy in patients with bilateral thyroid disease to confidently prevent bilateral RLN palsy. In fact, in $85 \%$ with known nerve injury and in $56 \%$ with negative IONM stimulation on the first side of dissection, the surgical strategy was changed with no resulting postoperative bilateral RLN palsy. This was in contrast to the $17 \%$ of patients with bilateral RLN palsy $(p<0.05)$ that occurred when surgeons were not aware of a preexisting or highly likely nerve injury on the first side of thyroid dissection [34••, 35].

Dralle et al. noticed that the growing appreciation that standardized IONM can prevent bilateral RLN palsies after signal loss on the initial side of resection may become increasingly relevant to malpractice litigation. Among German surgeons, $93.5 \%$ were willing to change their surgical strategy to prevent bilateral RLN palsy when unilateral LOS was present: $84.7 \%$ by discontinuing bilateral surgery and $8.8 \%$ by reducing the planned extent of surgery [36••].

According to Sadowski, even patients understand perfectly the benefit of staging thyroidectomy [37].

In view of the worldwide proliferation of IONM, it may become harder to defend future malpractice claims regarding bilateral RLN palsies, unless IONM was conducted on the initial side of resection according to international standards $[33,38,39 \bullet \bullet]$.

If the surgeon, despite the LOS to the first side, decides to continue to operate on the opposite side, he must justify the reason and clearly describe it in the medical records $[33,38,39 \bullet \bullet]$.

\section{Postoperative Management}

The position of the surgeon in cases of RLN damage is not easy to evaluate, because the correct analysis of the case requires precise assessment of the entire set of variables, including (a) evaluation of the individual case (i.e. thyroid disease); (b) the procedure (i.e. the fact that it is a high-risk operation); (c) the surgeon's position (experience, training, volume); (d) the surgeon's experience with IONM (standardized methodology); (e) the comprehensive description of the surgical procedure; and (f) traceability of the use of IONM in the specific case. The neurostimulation electromyographic documentation is essential to draw surgical conclusions that justify any intraoperative surgical deliberation and for forensic issues [39••].

The postoperative management of the patient undergoing thyroidectomy should include proper EMG documentation in the medical records. IONM converts muscle activity into recordable EMG signals that can be printed out. Documentation of the normal neurophysiologic signals of the RLNs may have a forensic function, permitting early differentiation between RLN-related and unrelated voice changes. 
Proper IONM documentation includes the printed or digital time traceable $V 1, R 1, R 2, V 2$ stimulations per side according to IONM international standards, together with preoperative and postoperative laryngoscopy findings (Table 2) $[1 \bullet \cdot, 9]$.

\section{Research}

Currently, IONM research can be classified into two categories: (a) animal experimental models and (b) clinical observational studies.

\section{Animal Experimental Models}

Translation of scientific discoveries into meaningful human applications, particularly novel surgical technologies such as IONM, requires development of suitable animal models. Experimental approaches can be used to test different anesthetic drugs, equipment settings, surgical procedures, new devices, and most importantly, the nerve injury models during IONM.

Several animal models, such as porcine/mini-pig [7, 25, 40-42, 43•• 44, 45], canine/dog [46, 47] and rabbit [48], have been used in recent IONM research. The porcine model is the most commonly used model, because pigs and humans have anatomical and physiological similarities, especially in the neck and larynx (Fig. 5). In addition, the use of the mini-pig is economical and does not raise the public concerns associated with the experimental use of primates, cats, and dogs, thus providing a cost-effective research model.

The current animal IONM research can be subdivided into four categories:

1. Safety and normative EMG data

$\mathrm{Wu}$ et al. [12] conducted a prospective porcine model (Fig. 5) evaluation to confirm the safety and investigate the

Table 2 Standardized IONM

Preoperative
Structured informed consent (discuss staging thyroidectomy)
$L 1$ : preoperative laryngoscope
Intraoperative
$V 1$ : stimulation of the $\mathrm{VN}$ before dissection
$R 1$ : stimulation of the RLN at first identification
$R 2$ : stimulation of the RLN after complete hemostasis
$V 2$ : stimulation of the $\mathrm{VN}$ at complete hemostasis
Postoperative
$L 2:$ postoperative laryngoscope
EMG documentation. Printed or digital time traceable $V 1, R 1$,
$R 2, V 2$ stimulations, including waveform morphology,
amplitude and latency

optimal intensity of electrical VN and RLN stimulation during IONM. They found no unusual electrophysiological or cardiopulmonary effects after continuous pulsatile VN and RLN stimulations for $10 \mathrm{~min}$.

2. Anesthetic perspective

Neuromuscular blocking agents (NMBAs) might diminish the EMG signal of the vocalis muscles during IONM. Lu [7, 41] conducted a porcine study to investigate the effects of NMBAs on IONM. They also compared the recovery profile of the laryngeal muscles between different agents and dosages, and translated the information into clinical applications.

3. Feasibility study of new approach or device

Witzel $\mathrm{K}$ et al. [42] used a porcine model to test the feasibility of IONM during transoral thyroid resection; Schneider et al. [25] used a pig model to test and confirm the feasibility of using a new vagal anchor electrode for continuous IONM ( $C$-IONM).

\section{Nerve injury study}

Different models of RLN injury during IONM, including transection $[43 \bullet \bullet, 44]$, clamping or crush [43••, 45-47], traction or compression [25, 43*0], and thermal injury $[25,43 \bullet \bullet, 48]$, have been studied, and their EMG tracings were recorded and correlated with the nerve injury.

\section{Clinical Observational Studies}

The current clinical IONM research can be subdivided into three categories:

\section{Anesthetic perspectives}

IONM requires good cooperation with the anesthesiologist. The following two issues are current areas of research interest.

\section{The Use of Muscle Relaxants (Neuromuscular Blocking Agents, NMBAs)}

The use of NMBAs in general anesthesia is essential for achieving clinically acceptable tracheal intubating conditions and preventing laryngeal trauma. NMBAs, however, can be a potential cause of false-negative responses during IONM. In the past 5 years, Chiang's study group has determined the feasibility of IONM after the administration of a nondepolarizing NMBA (a single dose of either rocuronium or atracurium) $[49,50]$. They also confirmed that a total of 1 ED95 of rocuronium $(0.3 \mathrm{mg} / \mathrm{kg})$ is an optimal dose for IONM [51]. 


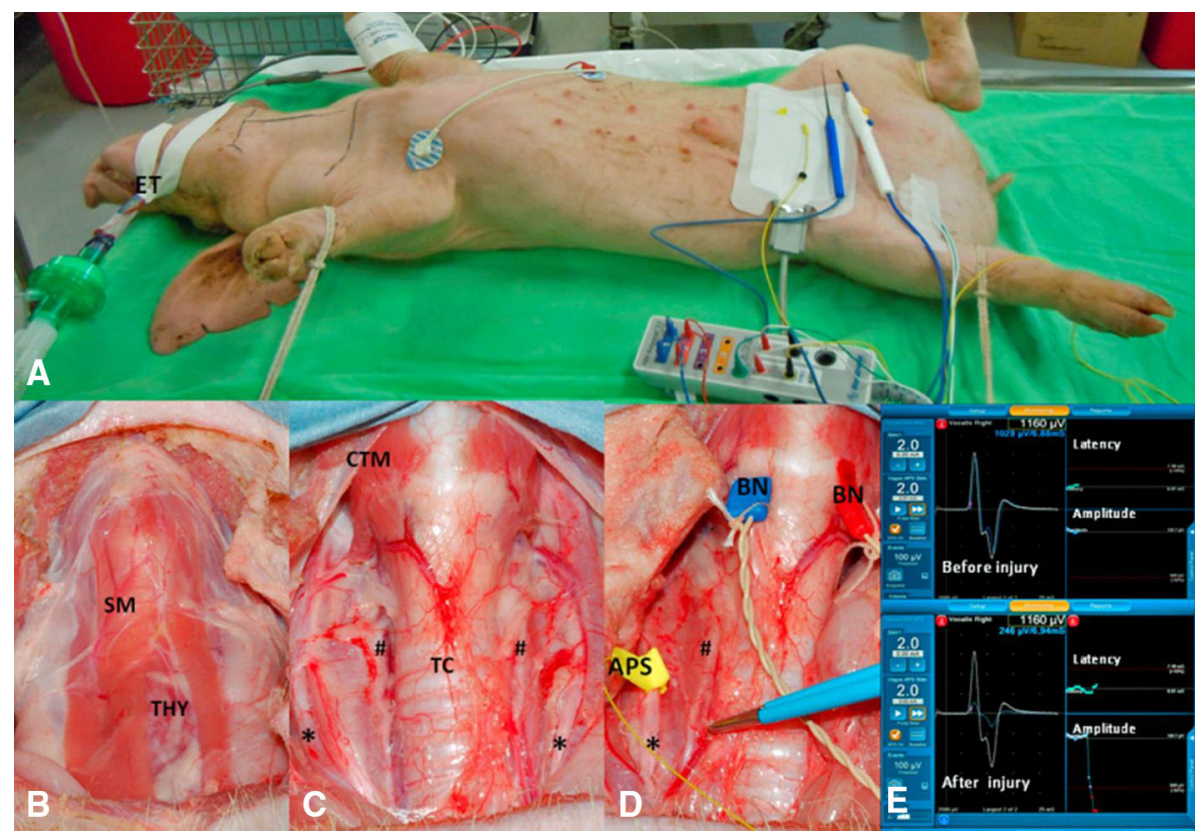

Fig. 5 Example of porcine animal model for IONM research. a A mini-pig was intubated with EMG endotracheal tube (ET) and prepared for IONM research. b Neck was opened. c Strap muscles (SC) and thyroid (THY) were removed to expose the trachea cartilage (TC), cricothyroid muscle (CTM), as well as the bilateral VNs (*) and RLNs (\#). d Bipolar needle (BN) electrodes can be inserted to vocalis

\section{The Placement of an EMG Endotracheal Tube}

Malposition of the endotracheal surface electrodes can result in equipment failure or unsuccessful monitoring, which could potentially give misleading information that might increase the risk of RLN injury. Chiang's study group [52] conducted a study to investigate the optimal depth of the EMG tube and concluded that the information would be a useful reference value for detecting the malposition of electrodes and adjusting the depth of the tube during the operation. Another study [8] found that the electrodes can be severely displaced when the patient's head position is changed from the neutral position for tracheal intubation to full extension for thyroid surgery. Therefore, the authors suggested checking the final electrode position routinely with laryngoscopy after patient positioning.

\section{Innovative application}

Many studies of innovative IONM applications have been published recently, including: (a) elucidation of surgical pitfalls and the mechanism of RLN injury [53••, 54]; (b) VN stimulation techniques for technical problem solving, recognizing any RLN lesions, precisely predicting RLN postoperative function [5, 6, 9, 55], and detecting a nonrecurrent laryngeal nerve [56-58]; (c) techniques for early RLN localization and identification (neural mapping) [59], identifying and handling the anatomical variations muscles for additional recording. Automated periodic stimulation (APS) electrode was put on the VN for CIONM. Various experiments can be tested on the RLN (in this photo, the thermal effect and injury on the RLN by bipolar cauterization was evaluated). e Real-time EMG tracings were recorded and correlated with the nerve experiments. (Photos from Dr. Chiang and Dr. Wu's animal Lab in Taiwan)

[60, 61••], and extensive RLN dissection [62]; (d) techniques for monitoring the external branch of the superior laryngeal nerve (EBSLN) [2••]; and (e) feasibility and devices for CIONM [25].

3. Normative Electrophysiological Data and Standardized Techniques

Normative intraoperative electrophysiological data for VN/RLN and EBSLN [63, 64] have been reported recently. "Normal" parameters are prerequisite for the interpretation of quantitative changes of intraoperative neuromonitoring during thyroid surgery to enable interpretation of influence on surgical strategy and prediction of postoperative vocal cord function $[1 \bullet \bullet, 63,64,65 \bullet \cdot]$. Lorenz [65••] analyzed systematic data of multicenter evaluation on quantitative intraoperative neuromonitoring parameters (amplitude, latency) of a total of 1,996 nerves. Median amplitude was significantly larger for the right versus left vagal nerve, latency was significantly longer for the left versus right vagal nerve, and duration of the left versus right vagal nerve was significantly longer. Age disparities were only present in the form of significantly higher amplitude in patients below 40 years of age. Regarding gender, there was significantly higher amplitude and smaller latency in women compared to men. Duration of surgery revealed a reduction of amplitude with operative time; contrarily, latency and signal duration remained stable. The type of 
underlying thyroid disease showed no influence on quantitative parameters of intraoperative neuromonitoring $[65 \bullet \bullet$.

\section{Conclusion}

High quality IONM is fundamental to optimizing surgical strategy that will yield the best patient outcomes as it relates to laryngeal function. The role of the surgeon is critical in obtaining an excellent quality of IONM. The surgeon, together with the anesthesiologist, should optimize the EMG signal, and in particular the $V 1$ signal, by means of appropriate verification of electrode materials and stimulation protocols, correct EMG tube position and proper use of induction and maintenance of anesthesia drugs. Members of the IONM Study Group have proposed a proper definitionllevel for $V 1$ amplitude $>500 \mathrm{mcV}[1 \bullet \bullet, 65 \bullet \cdot]$. $V 1$ signal is the prerequisite for the correct interpretation, diagnosis and verification of a functional intact RLN, for definition of a "significant" reduction of signal, "re-entry" signal (i.e. subsequent intraoperative EMG signal recovery and normal postoperative vocal cord function), LOS and again for the correct evaluation of the results. An unequivocal definition of normative EMG data is mandatory.

Conventional IONM is used to evaluate the function of RLN only at the moment of stimulation; using this technique, the nerve is still at risk of injury between two intermittent stimulations. When LOS is detected, the nerve has been injured. This leaves the surgeon without the opportunity to react immediately before LOS.

Consequently, several varieties of electrodes for VN stimulation have been designed for $C$-IONM, and they offer more seamless monitoring of the nerve's functional integrity along its entire course during surgery [25-28]. Some studies have reported that $C$-IONM is useful for detecting adverse EMG changes and preventing imminent RLN injury during the operation [25-28].

In an animal experiment [43••], $C$-IONM is actually a useful and objective tool to investigate the mechanism of RLN injury, particularly for thermal and traction injuries. The information of characteristic graded partial EMG change during acute RLN traction could be translated to clinical practice, and the substantial EMG decrease could be used as a warning criterion that would alert surgeons to immediately correct the surgical maneuver to prevent progressive nerve damage [43••].

Currently, conventional intermittent IONM remains more popular than $C$-IONM in clinical use; future studies are also needed to continuously develop innovative applications to make IONM a simple, safe, and surgeonfriendly procedure during thyroid and parathyroid operations.

\section{Compliance with Ethics Guidelines}

Conflict of Interest Gianlorenzo Dionigi, Che-Wei Wu, Davide Lombardi, Remo Accorona, Anna Bozzola, Hoon Yub Kim, Feng-Yu Chiang, Maurizio Bignami, Paolo Castelnuovo, and Piero Nicolai declare that they have no conflicts of interest.

Human and Animal Rights and Informed Consent This article does not contain any studies with human or animal subjects performed by any of the authors.

\section{References}

Papers of particular interest, published recently, have been highlighted as follows:

•- Of major importance

1. • Randolph GW, Dralle H, Abdullah H, et al. Electrophysiologic recurrent laryngeal nerve monitoring during thyroid and parathyroid surgery: international standards guideline statement. Laryngoscope. 2013;121 Suppl 1:S1-6. These guidelines are intended to improve the practice of neural monitoring of the inferior laryngeal nerve during thyroidectomy or parathyroidectomy, and to optimize clinical utility of this technique based on available evidence and consensus of experts.

2. • Barczyński M, Randolph GW, Cernea CR, et al. External branch of the superior laryngeal nerve monitoring during thyroid and parathyroid surgery: International Neural Monitoring Study Group standards guideline statement. Laryngoscope. 2013;123 Suppl 4:S1-14. These guidelines are intended to improve the practice of neural monitoring of the external branch of the superior laryngeal nerve during thyroidectomy or parathyroidectomy, and to optimize clinical utility of this technique based on available evidence and consensus of experts.

3. Dionigi G, Barczynski M, Chiang FY, et al. Why monitor the recurrent laryngeal nerve in thyroid surgery? J Endocrinol Invest. 2010;33(11):819-22.

4. Dionigi G, Kim HY, Wu CW, Lavazza M, et al. Vagus nerve stimulation for standardized monitoring: technical notes for conventional and endoscopic thyroidectomy. Surg Technol Int. 2013;17:XIII-X.

5. Wu CW, Dionigi G, Chen HC, et al. Vagal nerve stimulation without dissecting the carotid sheath during intraoperative neuromonitoring of the recurrent laryngeal nerve in thyroid surgery. Head Neck. 2012; doi:10.1002/hed.23154.

6. Dionigi G, Chiang FY, Rausei S, et al. Surgical anatomy and neurophysiology of the vagus nerve (VN) for standardised intraoperative neuromonitoring (IONM) of the inferior laryngeal nerve (ILN) during thyroidectomy. Langenbecks Arch Surg. 2010;395(7):893-9.

7. Lu IC, Chang PY, Hsu HT, et al. A comparison between succinylcholine and rocuronium on the recovery profile of the laryngeal muscles during intraoperative neuromonitoring of the recurrent laryngeal nerve: A prospective porcine model. Kaohsiung J Med Sci. 2013;29(9):484-7. doi:10.1016/j.kjms.2013. 01.002 Epub 2013 Apr 6.

8. Tsai CJ, Tseng KY, Wang FY, et al. Electromyographic endotracheal tube placement during thyroid surgery in neuromonitoring of recurrent laryngeal nerve. Kaohsiung J Med Sci. 2011;27(3): 96-101. doi:10.1016/j.kjms.2010.08.002 Epub 2011 Feb 22.

9. Chiang FY, Lee KW, Chen HC, et al. Standardization of intraoperative neuromonitoring of recurrent laryngeal nerve in thyroid operation. World J Surg. 2010;34(2):223-9. 
10. Dionigi G, Alesina PF, Barczynski M, et al. Recurrent laryngeal nerve injury in video-assistedthyroidectomy: lessons learned from neuromonitoring. Surg Endosc. 2012;26(9):2601-8.

11. Friedrich C, Ulmer C, Rieber F, et al. Safety analysis of vagal nerve stimulation for continuous nerve monitoring during thyroid surgery. Laryngoscope. 2012;122(9):1979-87.

12. Luginbuhl A, Schwartz DM, Sestokas AK, Cognetti D, Pribitkin E. Detection of evolving injury to the brachial plexus during transaxillary robotic thyroidectomy. Laryngoscope. 2012;122(1):110-5.

13. Imperatori A, Dionigi G, De Monte L, Conti V, Rotolo N. Cervicomediastinal schwannoma of the vagus nerve: resection with intraoperative nerve monitoring. Updates Surg. 2011;63(1):59-61.

14. Zhong D, Zhou Y, Li Y, et al. Intraoperative recurrent laryngeal nerve monitoring: a useful method for patients with esophageal cancer. Dis Esophagus. 2012;. doi:10.1111/j.1442-2050.2012. 01414.x.

15. Gelpke H, Grieder F, Decurtins M, Cadosch D. Recurrent laryngeal nerve monitoring during esophagectomy and mediastinal lymph node dissection. World J Surg. 2010;34(10):2379-82.

16. • Dralle H, Sekulla C, Lorenz K, Brauckhoff M, Machens A. Intraoperative monitoring of the recurrent laryngeal nerve in thyroid surgery. World J Surg 2008;32(7):1358-1366. Recurrent laryngeal nerve palsy rates (RLNPR) varied widely after thyroid surgery, ranging from 0 to $7.1 \%$ for transient RLN palsy to 0-11\% for permanent RLN palsy. These rates did not differ much from those reported for visual nerve identification without the use of IONM. Six studies with more than 100 nerves at risk (NAR) each evaluated RLNPR by contrasting IONM with visual nerve identification only. Recurrent laryngeal nerve palsy rates tended to be lower with IONM than without it, but this difference was not statistically significant. Six additional studies compared IONM findings with their corresponding postoperative laryngoscopic results. Those studies revealed high negative predictive values (NPV 92-100\%), but relatively low and variable positive predictive values (PPV 10-90\%) for IONM, limiting its utility for intraoperative RLN management.

17. • Barczyński M, Konturek A, Cichoń S. Randomized clinical trial of visualization versus neuromonitoring of recurrent laryngeal nerves during thyroidectomy. Br J Surg. 2009;96(3): 240-6. Nerve monitoring decreased the incidence of transient but not permanent RLN paresis compared with visualization alone, particularly in high-risk patients.

18. Timmermann W, Hamelmann WH, Thomusch O, et al. Effectiveness and results of intraoperative neuromonitoring in thyroid surgery. Statement of theInterdisciplinary Study Group on Intraoperative Neuromonitoring of Thyroid Surgery. Chirurg. 2004;75(9):916-22.

19. • Sancho JJ, Pascual-Damieta M, Pereira JA, et al. Risk factors for transient vocal cord palsy after thyroidectomy. $\mathrm{Br}$ J Surg.2008;95(8):961-7. Branched RLN suffer more surgical injuries and are twice as likely to be associated with vocal cord disfunction.

20. • Barczyński M, Konturek A, Pragacz K, Papier A, Stopa M, Nowak W. Intraoperative nerve monitoring can reduce prevalence of recurrent laryngeal nerve injury in thyroid reoperations: results of a retrospective cohort study. World J Surg. 2013. IONM decreased the incidence of transient RLN paresis in repeat thyroid operations compared with nerve visualization alone. The prevalence of permanent $R L N$ injury tended to be lower in thyroid reoperations with IONM.

21. Giordano D, Valcavi R, Thompson JB, Pedroni C, Renna L, Gradoni $\mathrm{P}$, et al. Complication of central neck dissection in patients with papillary thyroid carcinoma: results of a study on 1087 patients and review of the literature. Thyroid. 2012;22: 911-7.

22. Chiang FY, Lu CI, Tsai CJ, Hsiao PJ, Hsu CC, Wu CW. Does extensive dissection of recurrent laryngeal nerve during thyroid operation increase the risk of nerve injury? Evidence from the application of intraoperative neuromonitoring. Am J Otolaryngol. 2011;32:499-503.

23. Alesina PF, Rolfs T, Hommeltenberg S, Hinrichs J, Meier B, Mohmand $\mathrm{W}$, et al. Intraoperative neuromonitoring does not reduce the incidence of recurrent laryngeal nerve palsy in thyroid reoperations: results of a retrospective comparative analysis. World J Surg. 2012;36:1348-53.

24. Dionigi G, Van Slycke S, Boni L, Rausei S, Mangano A. Limits of neuromonitoring in thyroid surgery. Ann Surg. 2013;258(1): e1-2.

25. Schneider R, Przybyl J, Pliquett U, et al. A new vagal anchor electrode for real-time monitoring of the recurrent laryngeal nerve. Am J Surg. 2010;199(4):507-14.

26. Schneider R, Bures C, Lorenz K, et al. Evolution of nerve injury with unexpected EMG signal recovery in thyroid surgery using continuous intraoperative neuromonitoring. World J Surg. 2013;37(2):364-8

27. Schneider R, Randolph GW, Sekulla C, et al. Continuous intraoperative vagus nerve stimulation for identification of imminent recurrent laryngeal nerve injury. Head Neck. 2012;. doi:10.1002/ hed.23187.

28. Lamadé W, Ulmer C, Friedrich C, et al. Signal stability as key requirement for continuous intraoperative neuromonitoring. Chirurg. 2011;82(10):913-20.

29. Kandil E, Wassef SN, Alabbas H, Freidlander PL. Minimally invasive video-assisted thyroidectomy and parathyroidectomy with intraoperative recurrent laryngeal nerve monitoring. Int $\mathbf{J}$ Otolaryngol. 2009;2009:739798.

30. •• Dionigi G, Boni L, Rovera F, Bacuzzi A, Dionigi R Neuromonitoring and video-assisted thyroidectomy: a prospective, randomized case-control evaluation. Surg Endosc. 2009;23:9961003. This is the first VAT series with a standardized IONM technique. The technical feasibility and safety of IONM in selected patients seems acceptable. Neuromonitoring during VAT is effective in providing identification and function of laryngeal nerves. IONM enables surgeons to feel more comfortable with their approach to VAT. A reduction of rates for postoperative complications could not be demonstrated in the present study. Larger series are needed for further evaluation.

31. Lang BH, Wong KP. Feasibility on the use of intraoperative vagal nerve stimulation in gasless, transaxillary endoscopic, and robotic-assisted thyroidectomy. J Laparoendosc. 2011;21:911-7.

32. Dionigi G, Alesina PF, Barczynski M, et al. Recurrent laryngeal nerve injury in video-assisted thyroidectomy: lessons learned from neuromonitoring. Surg Endosc. 2012;26:2601-8.

33. Dionigi G, Frattini F. Staged thyroidectomy: time to consider intraoperative neuromonitoring as standard of care. Thyroid. 2013;23(7):906-8. doi:10.1089/thy.2013.0004 Epub 2013 Jun 21.

34. •• Goretzki PE, Schwarz K, Brinkmann J, Wirowski D, Lammers BJ. The impact of intraoperative neuromonitoring (IONM) on surgical strategy in bilateral thyroid diseases: is it worth the effort? World J Surg. 2010;34(6):1274-84. Failed IONM stimulation of the vagal or recurrent laryngeal nerve after resection of the first thyroid lobe is specific enough to reconsider the surgical strategy in patients with bilateral thyroid disease to surely prevent bilateral RLNP.

35. Melin M, Schwarz K, Lammers BJ, Goretzki PE. IONM-guided goiter surgery leading to two-stage thyroidectomy-indication and results. Langenbecks Arch Surg. 2013;398(3):411-8. doi:10. 1007/s00423-012-1032-7 Epub 2012 Nov 23.

36. • Dralle H, Sekulla C, Lorenz K, et al. Loss of the nerve monitoring signal during bilateral thyroid surgery. $\mathrm{Br} \mathrm{J}$ Surg. 2012;99(8):1089-95. RLN monitoring is now the standard of care during thyroidectomy in Germany. After LOS on the first side of resection in bilateral goitre, more than $90 \%$ of respondents 
declared their willingness to change the resection plan for the contralateral side to avoid the risk of bilateral recurrent laryngeal nerve palsy.

37. Sadowski SM, Soardo P, Leuchter I, Robert JH, Triponez F. Systematic use of recurrent laryngeal nerve neuromonitoring changes the operative strategy in planned bilateral thyroidectomy. Thyroid. 2013;23(3):329-33.

38. Angelos P. Ethical and medicolegal issues in neuromonitoring during thyroid and parathyroid surgery: a review of the recent literature. Curr Opin Oncol. 2012;24(1):16-21.

39. • Dralle H, Lorenz K, Machens A. Verdicts on malpractice claims after thyroid surgery: emerging trends and future directions. Head Neck. 2012;34(11):1591-6. The growing appreciation that standardized IONM can prevent bilateral RLN palsies after signal loss on the initial side of resection may become increasingly relevant to malpractice litigation.

40. Wu CW, Lu IC, Randolph GW, et al. Investigation of optimal intensity and safety of electrical nerve stimulation during intraoperative neuromonitoring of the recurrent laryngeal nerve: a prospective porcine model. Head Neck. 2010;32(10):1295-301.

41. Lu IC, Wang HM, Kuo YW, et al. Electromyographic study of differential sensitivity to succinylcholine of the diaphragm, laryngeal and somatic muscles: a swine model. Kaohsiung J Med Sci. 2010;26(12):640-6.

42. Witzel K, Benhidjeb T. Monitoring of the recurrent laryngeal nerve in totally endoscopic thyroid surgery. Eur Surg Res. 2009;43(2):72-6.

43. $\bullet \mathrm{Wu} \mathrm{CW}$, Dionigi G, Sun $\mathrm{H}$, et al. Intraoperative neuromonitoring for the early detection and prevention of RLN traction injury in thyroid surgery: a porcine model. Surgery. 2013. doi:10. 1016/j.surg.2013.08.015. IONM can be used as a tool for the early detection of adverse EMG changes that may alert surgeons to correct certain maneuvers immediately, to prevent irreversible nerve injury during the thyroid operation.

44. Birkholz T, Irouschek A, Labahn D, Klein P, Schmidt J. Electromyographic response persists after peripheral transection: endorsement of current concepts in recurrent laryngeal nerve monitoring in a porcine model. Langenbecks Arch Surg. 2010;395(2):121-5.

45. Moskalenko V, Huller M, Gasser M, et al. Investigation of the regeneration potential of the recurrent laryngeal nerve (RLN) after compression injury, using neuromonitoring. Langenbecks Arch Surg. 2009;394(3):469-74.

46. Scott AR, Chong PS, Brigger MT, Randolph GW, Hartnick CJ. Serial electromyography of the thyroarytenoid muscles using the NIM-response system in a canine model of vocal fold paralysis. Ann Otol Rhinol Laryngol. 2009;118(1):56-66.

47. Scott AR, Chong PS, Hartnick CJ, Randolph GW. Spontaneous and evoked laryngeal electromyography of the thyroarytenoid muscles: a canine model for intraoperative recurrent laryngeal nerve monitoring. Ann Otol Rhinol Laryngol. 2010;119(1):54-63.

48. Jiang $H$, Shen $H$, Jiang $D$, et al. Evaluating the safety of the Harmonic Scalpel around the recurrent laryngeal nerve. ANZ J Surg. 2010;80(11):822-6.

49. Chu KS, Wu SH, Lu IC, et al. Feasibility of intraoperative neuromonitoring during thyroid surgery after administration of nondepolarizing neuromuscular blocking agents. World J Surg. 2009;33(7):1408-13.

50. Chu KS, Tsai CJ, Lu IC, et al. Influence of nondepolarizing muscle relaxants on intraoperative neuromonitoring during thyroid surgery. J Otolaryngol Head Neck Surg. 2010;39(4):397-402.

51. Lu IC, Tsai CJ, Wu CW, et al. A comparative study between 1 and 2 effective doses of rocuronium for intraoperative neuromonitoring during thyroid surgery. Surgery. 2011;149(4):543-8.

52. Lu IC, Chu KS, Tsai CJ, et al. Optimal depth of NIM EMG endotracheal tube for intraoperative Neuromonitoring of the recurrent laryngeal nerve during thyroidectomy. World J Surg. 2008;32(9):1935-9.

53. • Chiang FY, Lu IC, Kuo WR, et al. The mechanism of recurrent laryngeal nerve injury during thyroid surgery: the application of intraoperative neuromonitoring. Surgery 2008;143(6):743-9. RLN injury during thyroidectomy or parathyroidectomy occurs intraoperatively significantly more often to a visually intact $R L N$ than to a transected nerve. The anterior motor branch of an RLN bifurcating near the ligament of Berry is particularly at risk of traction injury. Traction is the more common type of injury.

54. Snyder SK, Lairmore TC, Hendricks JC, Roberts JW. Elucidating mechanisms of recurrent laryngeal nerve injury during thyroidectomy and parathyroidectomy. J Am Coll Surg. 2008;206(1):123-30.

55. Dralle H, Sekulla C, Lorenz K, Brauckhoff M, Machens A. Intraoperative monitoring of the recurrent laryngeal nerve in thyroid surgery. World J Surg. 2008;32(7):1358-66.

56. Chiang FY, Lu IC, Tsai CJ, et al. Detecting and identifying nonrecurrent laryngeal nerve with the application of intraoperative neuromonitoring during thyroid and parathyroid operation. Am J Otolaryngol. 2012;33(1):1-5.

57. Donatini G, Carnaille B, Dionigi G. Increased detection of nonrecurrent inferior laryngeal nerve (NRLN) during thyroid surgery using systematic intraoperative neuromonitoring (IONM). World J Surg. 2013;37(1):91-3.

58. Brauckhoff M, Machens A, Sekulla C, Lorenz K, Dralle H. Latencies shorter than $3.5 \mathrm{~ms}$ after vagus nerve stimulation signify a nonrecurrent inferior laryngeal nerve before dissection. Ann Surg. 2011;253(6):1172-7.

59. Chiang FY, Lu IC, Chen HC, et al. Intraoperative neuromonitoring for early localization and identification of the recurrent laryngeal nerve during thyroid surgery. Kaohsiung J Med Sci. 2010;26(12):633-9.

60. Chiang FY, Lu IC, Chen HC, et al. Anatomical variations of recurrent laryngeal nerve during thyroid surgery: how to identify and handle the variations with intraoperative neuromonitoring. Kaohsiung J Med Sci. 2010;26(11):575-83.

61. • Serpell JW, Yeung MJ, Grodski S. The motor fibers of the recurrent laryngeal nerve are located in the anterior extralaryngeal branch. Ann Surg 2009;249(4):648-52. Extralaryngeal bifurcation of RLN is a common anatomical variant. By means of $I O N M$, the Author identified that the motor fibers of RLN are located in the anterior branch, for both adduction and abduction. Great care is therefore required following the presumed identification of the RLN, to ensure there is no unidentified anterior branch.

62. Chiang FY, Lu IC, Tsai CJ, et al. Does extensive dissection of recurrent laryngeal nerve during thyroid operation increase the risk of nerve injury? Evidence from the application of intraoperative neuromonitoring. Am J Otolaryngol. 2011;32(6):499-503.

63. Phelan E, Potenza A, Slough C, et al. Recurrent laryngeal nerve monitoring during thyroid surgery: normative vagal and recurrent laryngeal nerve electrophysiological data. Otolaryngol Head Neck Surg. 2012;147(4):640-6.

64. Potenza AS, Phelan EA, Cernea CR, et al. Normative Intraoperative Electrophysiologic Waveform Analysis of Superior Laryngeal Nerve External Branch and Recurrent Laryngeal Nerve in Patients Undergoing Thyroid Surgery. World J Surg. 2013;37(10):2336-42.

65. •• Lorenz K, Sekulla C, Schelle J, Schmeiss B, Brauckhoff M, Dralle H; German Neuromonitoring Study Group. What are normal quantitative parameters of intraoperative neuromonitoring (IONM) in thyroid surgery? Langenbecks Arch Surg.2010;395(7):901-9. Systematic data of multicenter evaluation on quantitative intraoperative neuromonitoring parameters revealed differences between left and right vagal nerves in regard to amplitude, latency and duration of signal, gender, and age. The nature of thyroid 
disease showed no significant influence on quantitative parameters of intraoperative neuromonitoring. This study presents for the first time collective data of a large series of nerves at risk in a multicenter setting. It seems that definitions of "normal" parameters are prerequisite for the interpretation of quantitative changes of intraoperative neuromonitoring during thyroid surgery, to enable interpretation of influence on surgical strategy and prediction of postoperative vocal cord function. 